\title{
Infantile Hypertrophic Pyloric Stenosis Following Repair of H-type Tracheoesophageal Fistula Repair and Post-operative Erythromycin Therapy
}

\author{
Natasha Merali ${ }^{1}$, Allison R Wilcox², Daniel P Croitoru' ${ }^{1,2}$, Reto M Baertschiger2* \\ ${ }^{1}$ Department of Pediatric General Surgery, Geisel School of Medicine at Dartmouth, Hanover, New Hampshire, United States of \\ America, ${ }^{2}$ Department of Surgery, Dartmouth-Hitchcock Medical Center, Lebanon, New Hampshire, United States of America \\ How to cite: Merali N, Wilcox AR, Croitoru DP, Baertschiger RM. Infantile hypertrophic pyloric stenosis following repair of \\ h-type tracheoesophageal fistula repair and post-operative erythromycin therapy. J Neonatal Surg. 2019;8:7. \\ This is an open-access article distributed under the terms of the Creative Commons Attribution License, which permits unrestricted use, \\ distribution, and reproduction in any medium, provided the original work is properly cited.
}

\begin{abstract}
We present a rare case of a newborn with H-type tracheoesophageal fistula (TEF) who developed pyloric stenosis postoperatively, 10 days after he was started on erythromycin to promote gastric emptying. We report this case to describe the relationship between H-type TEF repair, erythromycin therapy, and the development of infantile hypertrophic pyloric stenosis.
\end{abstract}

Key words: Erythromycin; Esophageal atresia; Infantile hypertrophic pyloric stenosis; H-type tracheoesophageal fistula

\section{CASE REPORT}

A $2398 \mathrm{~g}$ boy was born at home through spontaneous vaginal delivery to a G3P3 mother at 39-week gestational age. The pregnancy was complicated by conception with IUD still in place and subsequent inappropriate rise in progesterone. At 27-week gestation, the fetus was found to have intrauterine growth restriction and a two-vessel cord, without evidence of further anomalies. Within hours after birth, the baby was noted to have hypersalivation and gurgling noises and was transported to a local hospital for evaluation. Attempts at advancing a nasogastric tube were unsuccessful. X-ray showed the nasogastric tube curled in the upper esophagus, and the stomach distended with air (Figure 1). Esophageal atresia (EA) was suspected, and the patient was transferred to our tertiary care center for further care.

On admission to our hospital, a Replogle tube was able to be advanced into stomach with return of bile. On chest X-ray, several vertebral anomalies were noted in the midthoracic vertebrae, suggestive of a larger VACTERL association; however, subsequent workup did not reveal cardiac, renal, or anorectal anomalies. A barium swallow was obtained on the $2^{\text {nd }}$ day of life, demonstrating severe esophageal stenosis with near atresia, a large proximal pouch, and an associated tracheoesophageal fistula (TEF) at the level of the atresia. The patient was fed through a Replogle tube until undergoing operative repair at 10 days of age. He was confirmed to have an H-type fistula by bronchoscopy, and ligation division of the fistula was performed through a cervical approach without complications.

Postoperatively, he was started on famotidine to prevent esophageal strictures. Fluoroscopic swallow studies on post-operative days 6,13 , and 20 showed no evidence of persistent TEF but continued the presence of a tight stricture at the level of the anastomosis, with slowed and incomplete clearance past the stenotic segment. Serial esophageal balloon dilations were done on days of life $31,39,47$, and 57 , with a maximal dilation of $11 \mathrm{~mm}$. Over this period, the patient tolerated bolus enteral feeds through Replogle and some oral feeds, with inconsistent weight gain.

The patient gradually developed reflux, emesis, and thickened secretions following the third dilation. In an effort to treat these symptoms, erythromycin $5 \mathrm{mg} / \mathrm{kg}$ twice daily was added to his regimen at 48 days of age to promote gastric emptying. The dose was increased to $5 \mathrm{mg} / \mathrm{kg}$ every $6 \mathrm{~h}$ at 58 days old due to continued reflux; however, emesis and thick secretions did not 
resolve and increased in frequency. The erythromycin dose was subsequently decreased to $2.5 \mathrm{mg} / \mathrm{kg}$ every $6 \mathrm{~h}$. On day of life \#62, an abdominal and chest X-ray demonstrated a stomach distended with air and multiple dilated loops of bowel. An abdominal ultrasound confirmed the diagnosis of infantile hypertrophic pyloric stenosis (IHPS), demonstrating a pyloric wall thickness of $5 \mathrm{~mm}$ and channel length of $13 \mathrm{~mm}$ (Figure 2). The erythromycin was discontinued at this time.

On day 64, the patient underwent a pyloromyotomy and fifth esophageal balloon dilation to $11 \mathrm{~mm}$ without complication. Thereafter, the patient tolerated breast and bottle-feeding well accompanied with consistent weight gain and reduced emesis. By day 71, the patient was $>4 \mathrm{~kg}$ for the $1^{\text {st }}$ time. The Replogle feeding tube was removed on day 75 , and the patient was discharged the following day after 10 weeks in the neonatal intensive care unit. Today, the patient continues to demonstrate adequate growth and development but remains on the

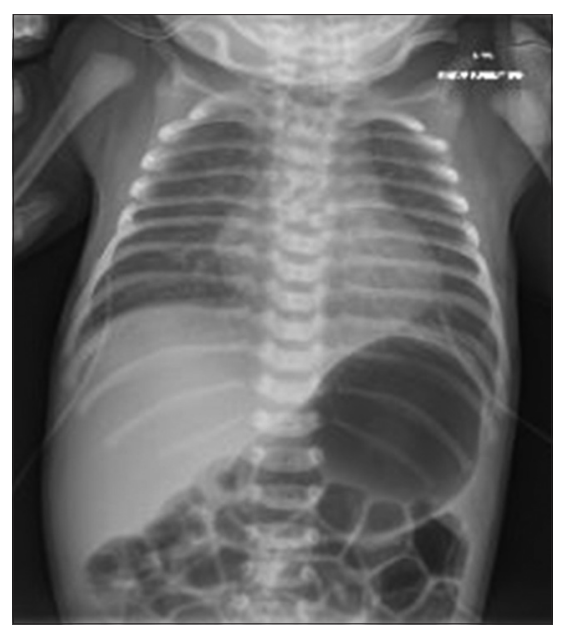

Figure 1: Abdominal X-ray demonstrating nasogastric tube curled in esophagus and stomach distended with air

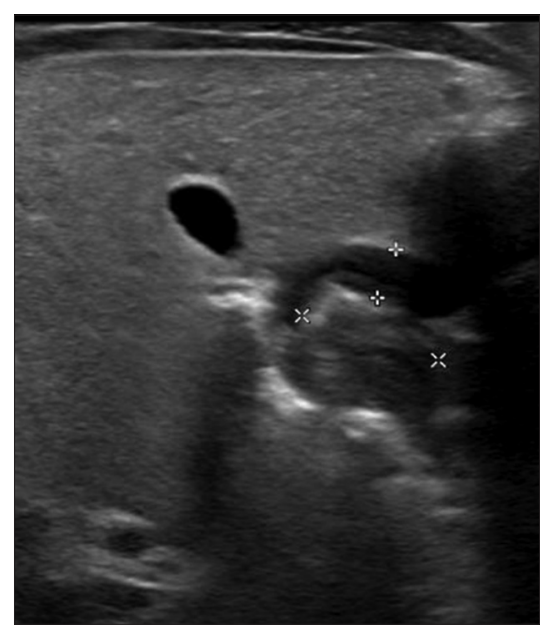

Figure 2: Ultrasound confirming hypertrophic pyloric stenosis, with measurement of $5 \mathrm{~mm}$ thickness and $13 \mathrm{~mm}$ length lower side of the growth curve. He occasionally experiences coughing post-feeds and will continue to have regular follow-up, and possible repeated esophageal dilations as indicated.

\section{DISCUSSION}

The prevalence of EA/TEF has remained stable over time, at approximately 2.5/10,000 births [1]. While EA/TEF is commonly associated with other malformations over $50 \%$ of the time, the incidence of IHPS in patients with $\mathrm{EA} / \mathrm{TEF}$ is not well established. Furthermore, the congenital $\mathrm{H}$-type $\mathrm{TEF}$, as found in our patient, is among the rarest subtypes, representing $<5 \%$ of all congenital TE malformations [2]. This rare combination of our patient's features invites us to consider the correlations between TEF, IHPS, and erythromycin therapy.

It has been found that patients with IHPS have a male predominance and genetic predisposition, including up to 30 times greater risk of IHPS in the sibling of an affected child [3]. Moreover, a retrospective study in 2014 of 267 patients in the Netherlands demonstrated a $7.5 \%$ incidence of IHPS in EA patients compared to a $0.25 \%$ incidence in the general population [4]. However, the association between operative repair of EA/TEA and development of IHPS is not well understood.

One possible explanation for IHPS following EA/TEA repair may be rooted in hormonal responses. Prior studies demonstrate that our body's "stress response" to surgery involves stimulation of the growth endocrine axis, leading to higher levels of growth hormone and insulin-like growth factor (IGF-1) [5]. Furthermore, expression of IGF-1 mRNA and its receptor in the circular muscle layer of the pylorus has been found to be higher in infants with IHPS, potentially making it a particularly susceptible region to post-surgical hormonal stress [6].

Of further interest in our patient is the potential role of erythromycin. Macrolide antibiotics, specifically erythromycin, are a well-known risk factor for the development of IHPS. Specifically, erythromycin use in the first 2 weeks of life has shown a 10-30-fold risk of HPS [4]. The mechanism for this association is that erythromycin stimulates motilin receptors, leading to strong contractions in the antrum and pylorus of the stomach that may promote pyloric hypertrophy [7]. Of note is that in our patient, pyloric stenosis was not detected on fluoroscopic swallow studies before erythromycin use in our patient. It is, therefore, possible that erythromycin therapy precipitated the development of IHPS in our patient, who may have already been predisposed to pyloric stenosis due to a combination of underlying genetic, anatomical, and hormonal elements post-EA/TEA repair.

We have reported a patient with a rare case of $\mathrm{H}$-type TEF who developed post-operative hypertrophic 
pyloric stenosis in the setting of erythromycin use. While patients who have undergone repair of EA/TEF appear to have a higher incidence of IHPS, it is generally low on the differential diagnosis for patients with post-operative reflux [8]. Given that, symptoms post-EA/TEF repair commonly overlap with symptoms of IHPS, persistent emesis in a newborn who has undergone TEF repair should raise concern and prompt evaluation for the development of IHPS to prevent progression of the condition and delay of a pyloromyotomy. Furthermore, since EA/TEF may be related to the development of IHPS, erythromycin therapy should be used with caution in these patients due to a conceivable heightened risk. Finally, further, research is needed to understand the indication of erythromycin, specifically in the treatment of post-EA/TEF gastroesophageal reflux.

\section{REFERENCES}

1. Nassar N, Leoncini E, Amar E, Arteaga-Vázquez J, Bakker MK, Bower C, et al. Prevalence of esophageal atresia among 18 international birth defects surveillance programs. Birth Defects Res A Clin Mol Teratol. 2012;94:893-9.
2. Al-Salem AH, Mohaidly MA, Al-Buainain HM, Al-Jadaan S, Raboei E. Congenital H-type tracheoesophageal fistula: A national multicenter study. Pediatr Surg Int. 2016;32:487-91.

3. Panteli C. New insights into the pathogenesis of infantile pyloric stenosis. Pediatr Surg Int. 2009;25:1043-52.

4. van Beelen NW, Mous DS, Brosens E, de Klein A, van de Ven CP, Vlot $J$, et al. Increased incidence of hypertrophic pyloric stenosis in esophageal atresia patients. Eur J Pediatr Surg. 2014;24:20-4.

5. Floros T, Philippou A, Bardakostas D, Mantas D, Koutsilieris M. The growth endocrine axis and inflammatory responses after laparoscopic cholecystectomy. Hormones (Athens). 2016;15:73-80.

6. Oshiro K, Puri P. Increased insulin-like growth factor and platelet-derived growth factor system in the pyloric muscle in infantile hypertrophic pyloric stenosis. J Pediatr Surg. 1998;33:378-81.

7. El-Gohary Y, Abdelhafeez A, Paton E, Gosain A, Murphy AJ. Pyloric stenosis: An enigma more than a century after the first successful treatment. Pediatr Surg Int. 2018;34:21-7.

8. Tovar JA, Fragoso AC. Gastroesophageal reflux after repair of esophageal atresia. Eur J Pediatr Surg. 2013;23:175-81. 